\title{
K2-60b and K2-107b. A Sub-Jovian and a Jovian Planet from the K2 Mission
}

\author{
Philipp Eigmüller ${ }^{1}$, Davide Gandolfi ${ }^{2,3}$, Carina M. Persson ${ }^{4}$, Paolo Donati ${ }^{5}$, Malcolm Fridlund ${ }^{4,6}$, Szilard Csizmadia ${ }^{1}$, \\ Oscar Barragán $^{2}$, Alexis M. S. Smith ${ }^{1}$, Juan Cabrera ${ }^{1}$, Judith Korth ${ }^{7}$, Sascha Grziwa ${ }^{7}$, Jorge Prieto-Arranz ${ }^{8,9}$, David Nespral ${ }^{8,9}$, \\ Joonas Saario $^{10}$, William D. Cochran ${ }^{11}$, Felice Cusano, Hans J. Deeg ${ }^{8,9}$, Michael Endl ${ }^{11}$, Anders Erikson ${ }^{1}$, Eike W. Guenther ${ }^{12}$, \\ Artie P. Hatzes ${ }^{12}$, Martin Pätzold ${ }^{7}$, and Heike Rauer ${ }^{1,13}$ \\ ${ }^{1}$ Institute of Planetary Research, German Aerospace Center, Rutherfordstrasse 2, D-12489 Berlin, Germany \\ ${ }^{2}$ Dipartimento di Fisica, Universitá di Torino, via P. Giuria 1, I-10125 Torino, Italy \\ ${ }^{3}$ Landessternwarte Königstuhl, Zentrum für Astronomie der Universität Heidelberg, Königstuhl 12, D-69117 Heidelberg, Germany \\ ${ }^{4}$ Department of Earth and Space Sciences, Chalmers University of Technology, Onsala Space Observatory, SE-439 92 Onsala, Sweden \\ ${ }^{5}$ INAF-Osservatorio Astronomico di Bologna, Via Ranzani, 1, I-40127, Bologna, Italy \\ ${ }^{6}$ Leiden Observatory, University of Leiden, PO Box 9513, 2300 RA, Leiden, The Netherlands \\ ${ }^{7}$ Rheinisches Institut für Umweltforschung an der Universität zu Köln, Aachener Strasse 209, D-50931 Köln, Germany \\ ${ }^{8}$ Instituto de Astrofísica de Canarias, E-38205 La Laguna, Tenerife, Spain \\ ${ }^{9}$ Departamento de Astrofísica, Universidad de La Laguna, E-38206 La Laguna, Spain \\ ${ }^{10}$ Nordic Optical Telescope, Apartado 474, E-38700, Santa Cruz de La Palma, Spain \\ ${ }^{11}$ Department of Astronomy and McDonald Observatory, University of Texas at Austin, \\ 2515 Speedway, Stop C1400, Austin, TX 78712, USA \\ 12 Thüringer Landessternwarte Tautenburg, Sternwarte 5, D-07778 Tautenberg, Germany \\ ${ }^{13}$ Center for Astronomy and Astrophysics, TU Berlin, Hardenbergstr. 36, D-10623 Berlin, Germany \\ Received 2016 November 11; revised 2017 January 26; accepted 2017 January 27; published 2017 February 27
}

\begin{abstract}
We report the characterization and independent detection of K2-60b, as well as the detection and characterization of K2-107b, two transiting hot gaseous planets from the K2 space mission. We confirm the planetary nature of the two systems and determine their fundamental parameters combining the K2 time-series data with FIES@NOT and HARPS-N@TNG spectroscopic observations. K2-60b has a radius of $0.683 \pm 0.037 R_{\text {Jup }}$ and a mass of $0.426 \pm 0.037 M_{\text {Jup }}$ and orbits a G4 V star with an orbital period of $3.00267 \pm 0.00006$ days. K2-107b has a radius of $1.44 \pm 0.15 R_{\mathrm{Jup}}$ and a mass of $0.84 \pm 0.08 M_{\mathrm{Jup}}$ and orbits an F9 IV star every $3.31392 \pm 0.00002$ days. K2-60b is among the few planets at the edge of the so-called "desert" of short-period sub-Jovian planets. K2$107 \mathrm{~b}$ is a highly inflated Jovian planet orbiting an evolved star about to leave the main sequence.
\end{abstract}

Key words: planetary systems - planets and satellites: detection - planets and satellites: gaseous planets planets and satellites: individual (K2-60 b, K2-107 b)

\section{Introduction}

More than 3500 exoplanets have been discovered over the last 25 years (Schneider et al. 2011). ${ }^{14}$ This has allowed us to compare the observed exoplanet populations with formation theories and evolutionary models (e.g., Mordasini et al. 2009a, 2009b, 2012; Alibert et al. 2011). One of the highly discussed topics in exoplanetary science is the so-called "sub-Jovian desert", which describes a significant dearth of exoplanets with masses lower than $\sim 300$ Earth masses and orbital periods below two to four days (Szabó \& Kiss 2011; Beaugé \& Nesvorný 2013; Mazeh et al. 2016).

Whereas lower mass planets get reduced in size due to photo-evaporation (Lundkvist et al. 2016), hot Jovian planets, more massive than Jupiter and with orbital periods below four days, tend to be inflated. A detailed empirical study of these radius anomalies was conducted by Laughlin et al. (2011) who found a clear correlation between the planets' orbit-averaged effective temperatures and the observed inflation. Laughlin et al. (2011) suggested that the Ohmic heating might account for the observed inflation. This effect could influence the upper border of this "desert" related to the radius. However, as Mazeh et al. (2016) showed, this desert is also present in the mass regime. Recent theoretical studies on planet formation give additional explanations for the

\footnotetext{
${ }^{14}$ From http://www.exoplanet.eu, as of 2016 September 26.
}

boundaries of the desert using in situ formation (Batygin et al. 2016), as well as planet migration theories (Matsakos \& Königl 2016). Unfortunately, the lack of well characterized planets in the regime close to the sub-Jovian desert does not allow us at the moment to give strict constraints on its border. The upper border seems to be well defined due to the large amount of planets detected with ground-based transit surveys, but, as a comparison with Kepler planets shows, the detection bias of these ground-based surveys does not allow us to extrapolate the upper border of the sub-Jovian desert to a regime for planets smaller than $0.8 R_{\text {Jup }}$. The number of well characterized Kepler planets on the other hand are also very limited. A better empirical definition of the sub-Jovian desert and its boundaries might allow further constraints to be placed on planet formation and evolution models.

Here we report our results on K2-60b and K2-107b, which are both short-period planets with orbital periods of approximately three days. The small mass and size of K2-60b puts this planets close to the sub-Jovian desert and thus might help us to better restrict its boundaries in the future. K2-107b, on the other hand, is a highly inflated planet. It is a member of the inflated hot Jupiters, but it is only one of the few known to orbit a sub-giant host star.

Planet K2-60b has recently been reported as a planet candidate by Crossfield et al. (2016) and validated using high-resolution imaging by Schmitt et al. (2016). However, the 

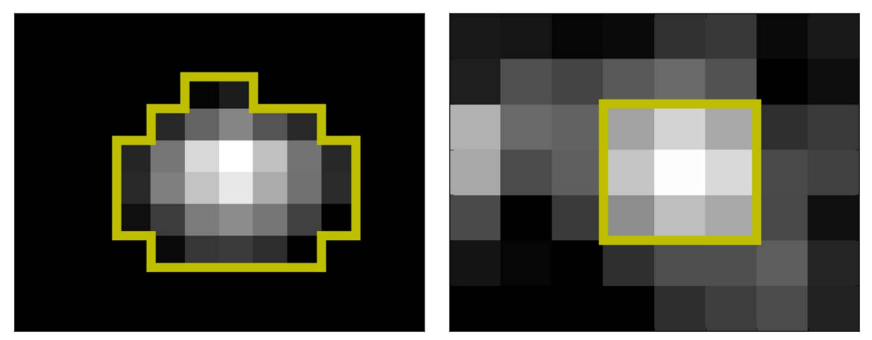

Figure 1. K2 stamps of K2-60 (left) and K2-107 (right). The yellow lines represent the adopted photometric apertures. The pixel scale of the Kepler spacecraft is 3 ". 98 per pixel. The stamp of K2-60 has a size of $12 \times 10$ pixels, whereas the stamp of K2-107 has a size of $8 \times 7$ pixels. The gray scale represents the counts per pixel.
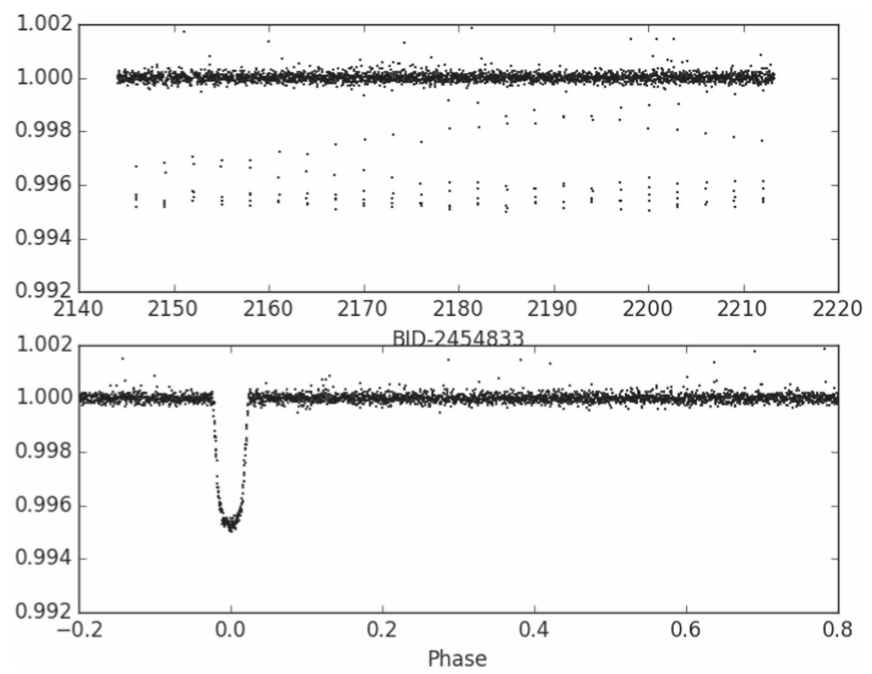

Figure 2. Corrected and normalized light curve of K2-60. The upper plot shows the normalized light curve over time. The lower plot displays the phase folded light curve.

planet has not been characterized before in terms of mass and bulk density.

\section{Observations}

\subsection{K2 Photometry and Transit Detection}

The Kepler space observatory, launched in 2009, was designed to provide precise photometric monitoring of over 150,000 stars in a single field and to detect transiting Earthsized planets with orbital periods of up to one year (Borucki et al. 2010). In the spring of 2013, after four years of operation in space, the failure of the second reaction wheel caused the end of the mission, as it was no longer possible to precisely point the telescope. At the end of 2013, the operation of the Kepler space telescope re-started with a new concept that uses the remaining reaction wheels, the spacecraft thrusters, and solar wind pressure to point the telescope. The new mission, called K2 (Howell et al. 2014), enables the continued use of the Kepler spacecraft with limited pointing accuracy. In contrast to the Kepler mission, $\mathrm{K} 2$ observes different fields located along the ecliptic for a duration of about three consecutive months per field. EPIC 206038483 (K2-60) was observed by the K2 mission in campaign 3 from 2014 November until 2015 February. EPIC 216468514 (K2-107) was observed in campaign 7, between 2015 October and December.

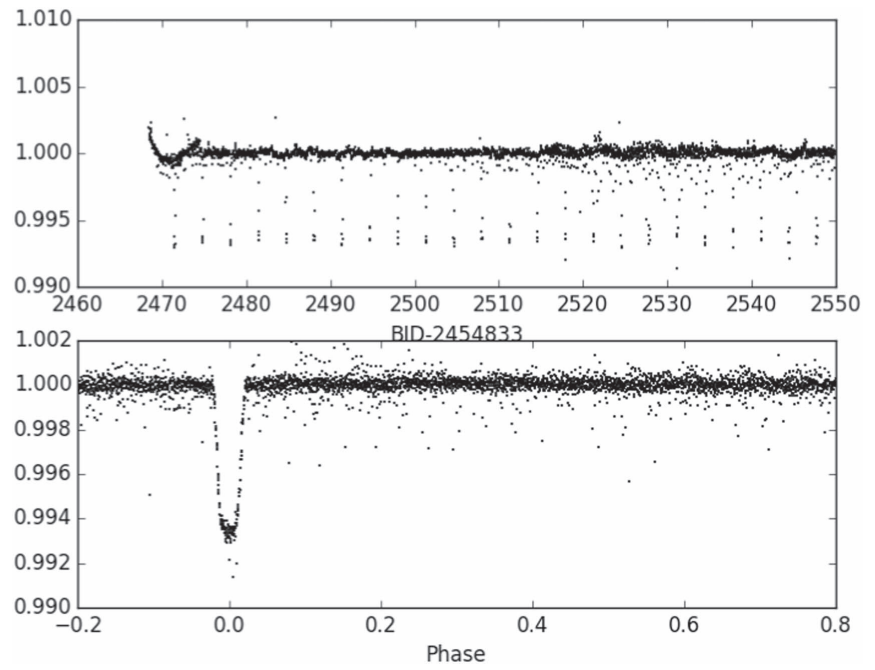

Figure 3. Corrected and normalized light curve of K2-107. Notation as in Figure 2.

To detect transit signals in $\mathrm{K} 2$ campaigns 3 and 7, we used the light curves extracted by Vanderburg \& Johnson (2014) from the K2 data. We used the same algorithms and vetting tools described in Cabrera et al. (2012), Grziwa et al. (2012), and Grziwa \& Pätzold (2016b). These algorithms have been largely used by our team to detect and confirm planets in other K2 fields (Barragán et al. 2016; Grziwa et al. 2016a; Johnson et al. 2016; Smith et al. 2016). For the modeling of the transit light curves, we used our own optimized photometry employing a similar approach as in Vanderburg \& Johnson (2014), which allowed us to reduce strong systematics by choosing optimal segment sizes when splitting the light curve for decorrelation. The photometry was performed using a fixed aperture for each object as shown in Figure 1. For K2-60, we selected an aperture of 33 pixels as the star is isolated. In the case of $\mathrm{K} 2-107$, this target is in a field that is close to the galactic center and thus very crowded. We minimized the contamination effects arising from nearby sources by using a fixed aperture of only 9 pixels (Figure 1). As in the pipeline of Kepler and Vanderburg \& Johnson (2014), each light curve was split into segments to remove correlated noise. The length of these segments influences the quality of de-correlation. We found an optimal size for the segments to be twice the orbital period of the planet. This way we avoided splitting the light curve within any transit signal. These short segments were individually de-correlated against the relative motion of the star, given in the POS_CORR columns. ${ }^{15}$ To remove long-term trends, we de-correlated these segments also in the time domain after ruling out the existence of ellipsoidal variations in the phase folded light curve that might hint at eclipsing binary systems. The resulting light curves, in the time domain and phase folded, are shown in Figures 2 and 3.

\subsection{High Dispersion Spectroscopy}

We acquired five and eight high-resolution spectra $(R \approx 67,000)$ of $\mathrm{K} 2-60$ and $\mathrm{K} 2-107$ with the the Flbre-fed Échelle Spectrograph (FIES; Frandsen \& Lindberg 1999; Telting et al. 2014) between 2016 June and September. FIES is mounted

\footnotetext{
$\overline{15}$ Due to the strong correlation between POS_CORR1 and POS_CORR2, it was sufficient to use POS_CORR1 for de-correlation.
} 
Table 1

FIES and HARPS-N RV Measurements of K2-60 and K2-107

\begin{tabular}{lccrl}
\hline \hline $\begin{array}{l}\text { BJD } \\
-2,450,000\end{array}$ & $\begin{array}{c}\mathrm{RV} \\
\left(\mathrm{km} \mathrm{s}^{-1}\right)\end{array}$ & $\begin{array}{c}\sigma_{\mathrm{RV}} \\
\left(\mathrm{km} \mathrm{s}^{-1}\right)\end{array}$ & $\begin{array}{c}\text { BIS } \\
\left(\mathrm{km} \mathrm{s}^{-1}\right)\end{array}$ & Instr. \\
\hline K2-60 & & & & \\
7568.72048 & -45.505 & 0.012 & 0.007 & FIES \\
7569.72143 & -45.394 & 0.024 & -0.006 & FIES \\
7570.71124 & -45.490 & 0.012 & 0.022 & FIES \\
7577.71171 & -45.532 & 0.012 & 0.014 & FIES \\
7578.64730 & -45.422 & 0.030 & 0.018 & FIES \\
7585.67140 & -45.324 & 0.010 & -0.036 & HARPS-N \\
7586.68055 & -45.362 & 0.006 & -0.023 & HARPS-N \\
7587.70160 & -45.260 & 0.007 & 0.007 & HARPS-N \\
\hline K2-107 & & & & \\
7565.58753 & -8.276 & 0.025 & 0.069 & FIES \\
7566.56965 & -8.404 & 0.023 & 0.012 & FIES \\
7567.57489 & -8.438 & 0.016 & 0.025 & FIES \\
7568.59817 & -8.272 & 0.021 & 0.044 & FIES \\
7570.54490 & -8.465 & 0.017 & 0.043 & FIES \\
7628.44921 & -8.273 & 0.029 & -0.007 & FIES \\
7637.39240 & -8.392 & 0.016 & 0.004 & FIES \\
7640.40979 & -8.429 & 0.016 & 0.035 & FIES \\
\hline
\end{tabular}

at the $2.56 \mathrm{~m}$ Nordic Optical Telescope (NOT) of Roque de los Muchachos Observatory (La Palma, Spain). We adopted the same observing strategy as in Buchhave et al. (2010) and Gandolfi et al. (2015), i.e., we bracketed each science observation with long exposed ThAr spectra $\left(T_{\exp } \approx 35 \mathrm{~s}\right)$. The exposure time was set to 1800-3600 s-according to sky conditions and scheduling constraints-leading to a signal-to-noise ratio $(\mathrm{S} / \mathrm{N})$ of $25-35$ per pixel at $5500 \AA$. The FIES data were reduced using standard IRAF and IDL procedures. Radial velocity measurements were extracted via multi-order cross-correlation with the RV standard stars HD 50692 and HD 182572 (Udry et al. 1999) observed with the same instrument set-up as the target stars.

We also took three additional high-resolution spectra of K2-60 in 2016 July with the HARPS-N spectrograph $(R \approx 115,000$; Cosentino et al. 2012) mounted at the $3.58 \mathrm{~m}$ Telescopio Nazionale Galileo (TNG) at Roque de los Muchachos Observatory (La Palma, Spain). The exposure times were set to $1200-1500 \mathrm{~s}$ leading to an $\mathrm{S} / \mathrm{N}$ of $15-20$ per pixel at $5500 \AA$ for the extracted spectra. We used the second fiber to monitor the Moon background and reduced the data with the HARPS-N dedicated pipeline. Radial velocities were extracted by crosscorrelating the extracted spectra with a G2 numerical mask (Baranne et al. 1996; Pepe et al. 2002).

The FIES and HARPS-N RVs and their uncertainties are listed in Table 1. Time stamps are given in barycentric Julian day in barycentric dynamical time $\left(\mathrm{BJD}_{\mathrm{TDB}}\right)$. Table 1 also reports the bisector span (BIS) of the cross-correlation function (CCF). Following Toner \& Gray (1988) and Queloz et al. (2001), we define the CCF BIS as the difference between the average velocities in two different regions of the bisector, one at the top and one close to the bottom.

We searched for possible correlation between the RV and BIS measurements that might unveil activity-induced RV variations and/or the presence of blended eclipsing binary systems (Queloz et al. 2001). The Pearson correlation coefficient between the RV and BIS measurements of K2-60 is 0.11 with a $p$-value of 0.79 . For K2-107, the Pearson correlation coefficient is 0.10 with a $p$-value of 0.81 . Adopting

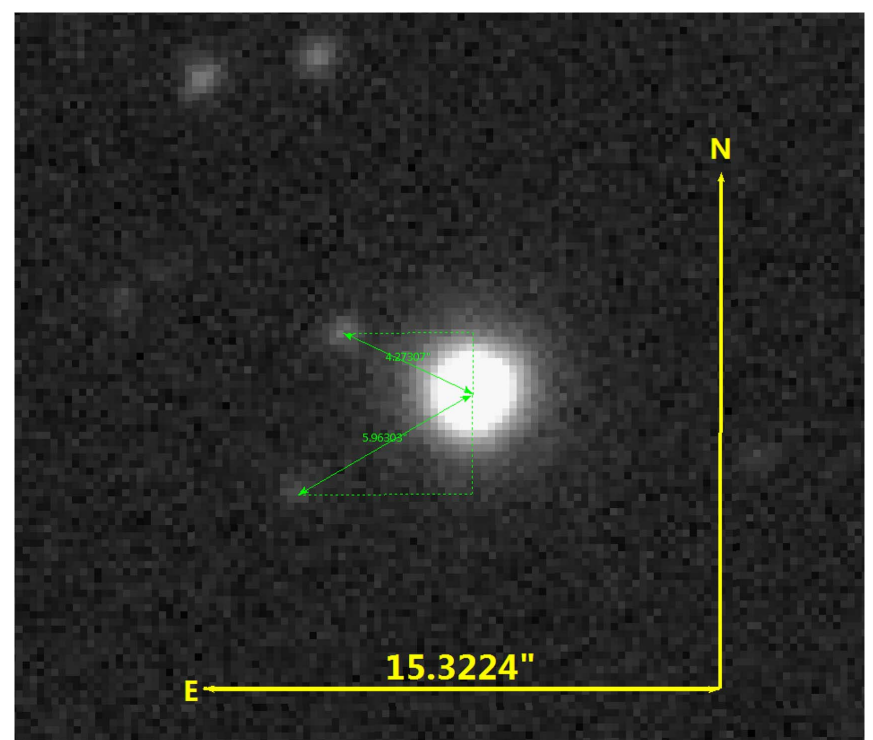

Figure 4. ALFOSC@NOT R-band image of K2-107. We can resolve sources as close as $2^{\prime \prime}$ to our target star. K2-107 and its two contaminants are marked with green circles.

the threshold of 0.05 for the $p$-value confidence level (Lucy \& Sweeney 1971), the lack of significant correlations between the $\mathrm{RV}$ and BIS measurements of both stars further confirm that the observed Doppler variations are induced by the orbiting planets.

\subsection{Imaging}

Imaging with spatial resolution higher than that of $\mathrm{K} 2$ is used to detect potential nearby eclipsing binaries that could mimic planetary transit-like signals. It also enables us to measure the fraction of contaminating light arising from potential unresolved nearby sources whose light leaks into the photometric mask of K2, thus diluting the transit signal. Schmitt et al. (2016) observed K2-60 using the adaptive optics facility at the KECK telescope. They excluded faint contaminant stars as close as 0 "' 25 up to 4 mag fainter than the target star.

We observed K2-107 on 2016 September 13 (UT) with the ALFOSC camera mounted at the NOT. We used the Johnson's standard $R$-band filter and acquired 16 images of $6 \mathrm{~s}$ and 2 images of $20 \mathrm{~s}$. The data were bias subtracted and flat-fielded using dusk sky flats. The co-added $6 \mathrm{~s}$ ALFOSC exposures are shown in Figure 4. We detected two nearby faint stars located 4." 3 northeast and 6." 0 southeast of K2-107. They are 6.3 and $6.5 \mathrm{mag}$ fainter than the target and fall inside the photometric aperture that we used to extract the light curve of K2-107 from the K2 images. We measured a contribution of $0.005 \pm 0.001$ to the total flux, by contaminating sources for K2-107. Our observations exclude additional contaminants out to a separation of $2^{\prime \prime}$ and up to 6 mag fainter than the target. We compared our findings with the first data release from GAIA (Gaia Collaboration et al. 2016) and found a contamination factor of 0.0043 , in agreement with our estimate. No additional sources are present in the GAIA catalog (Lindegren et al. 2016) within a radius of $10^{\prime \prime}$. The resolving power of GAIA is well below $1^{\prime \prime}$. 
Table 2

Effective Temperature, Surface Gravity, and Metallicity from Different Spectral Analysis Methods

\begin{tabular}{lccccc}
\hline \hline Method & & $\mathrm{K} 2-60$ & & $\mathrm{~K} 2-107$ \\
$\log g_{\star}(\mathrm{cgs})$ & $T_{\text {eff }}(\mathrm{K})$ & $6.95 \pm 0.10$ \\
\hline Method 1 & $5480 \pm 85$ & $4.05 \pm 0.15$ & $-0.10 \pm 0.10$ & $6050 \pm 110$ & $0.08 \pm 0.06$ \\
Method 2 & $5350 \pm 90$ & $3.95 \pm 0.10$ & $-0.10 \pm 0.08$ & $5970 \pm 100$ & $4.30 \pm 0.15$ \\
Method 3 & $5625 \pm 115$ & $4.22 \pm 0.07$ & $0.24 \pm 0.15$ & $6080 \pm 150$ & $3.95 \pm 0.05$ \\
\hline
\end{tabular}

\section{Analysis}

\subsection{Spectral Analysis}

We derived the spectroscopic parameters of K2-60 and K2-107 from the co-added spectra used to extract the RVs of the stars (Section 2.2). The stacked FIES and HARPS-N have an $\mathrm{S} / \mathrm{N}$ of 62 and 32 per pixel at $5500 \AA$; the co-added FIES data of K2-107 have an S/N of 76 per pixel at $5500 \AA$. The analysis was carried out in three independent ways.

The first technique uses ATLAS 9 model spectra (Castelli \& Kurucz 2004) to fit spectral features that are sensitive to different photospheric parameters. We adopt the calibration equations of Bruntt et al. (2010) and Doyle et al. (2014) to determine the microturbulent $\left(V_{\text {mic }}\right)$ and macroturbulent $\left(V_{\mathrm{mac}}\right)$ velocities. We mainly used the wings of the $\mathrm{H}_{\alpha}$ and $\mathrm{H}_{\beta}$ lines to estimate the effective temperature $\left(T_{\text {eff }}\right)$, and the $\operatorname{Mg}$ I 5167, 5173, and $5184 \AA$, Ca I 6162 and $6439 \AA$, and the Na I D lines to determine the surface gravity $\log g_{\star}$. We simultaneously fit different spectral regions to measure the metal abundance $[\mathrm{M} / \mathrm{H}]$. The projected rotational velocity $v \sin i_{\star}$ was determined by fitting the profile of many isolated and unblended metal lines.

For the second method, microturbulent $\left(V_{\text {mic }}\right)$ and macroturbulent $\left(V_{\mathrm{mac}}\right)$ velocities, as well as the projected stellar rotational velocity $v \sin i_{\star}$ were determined as described above. For the spectral analysis, the second method relies on the use of the package SME (Spectroscopy Made Easy, where we used version 4.43) (Valenti \& Piskunov 1996; Valenti \& Fischer 2005). SME calculates, using a grid of models (we used the Atlas 12) for a set of given stellar parameters, synthetic spectra of stars and fits them to the observed high-resolution spectra using a $\chi$-square minimizing procedure.

The third method uses the equivalent width (EW) method to derive stellar atmospheric parameters: (i) $T_{\text {eff }}$ is measured by eliminating trends between the abundance of the chemical elements and the respective excitation potentials; (ii) $\log g_{\star}$ is derived by assuming the ionization equilibrium condition, i.e., requiring that for a given species, the same abundance (within the uncertainties) is obtained from lines of two ionization states (typically, neutral and singly ionized lines); (iii) microturbulent velocity is set by minimizing the slope of the relationship between abundance and the logarithm of the reduced EWs. We measured the EWs using the DOOp program Cantat2014, a wrapper of DAOSPEC (Stetson \& Pancino 2008). We derived the photospheric parameters with the program FAMA (Magrini et al. 2013), a wrapper of MOOG (Sneden et al. 2012). The adopted atomic parameters are the public version of those prepared for the Gaia-ESO Survey (Heiter et al. 2015) and based on the VALD3 data (Ryabchikova et al. 2011). We typically used $200 \mathrm{Fe}$ I lines and $10 \mathrm{Fe}$ II lines for the determination of stellar parameters.

The three methods provide consistent results within two sigma (see Table 2). The final adopted values are the weighted
Table 3

Main Identifiers, Coordinates, Magnitudes, and Spectroscopic Parameters of Both Systems

\begin{tabular}{lccc}
\hline \hline Parameter & $\mathrm{K} 2-60$ & $\mathrm{~K} 2-107$ & Unit \\
\hline RA & $22^{\mathrm{h}} 34^{\mathrm{m}} 25^{\mathrm{s}} \cdot 49$ & $18^{\mathrm{h}} 59^{\mathrm{m}} 56^{\mathrm{s}} 49$ & $\mathrm{hr}$ \\
DEC & $-13^{\circ} 43^{\prime} 54^{\prime \prime} 13$ & $-22^{\circ} 17^{\prime} 36^{\prime \prime} 25$ & $\mathrm{deg}$ \\
2MASS ID & $22342548-$ & $18595649-$ & $\cdots$ \\
& 1343541 & 2217363 & \\
EPIC ID & 206038483 & 216468514 & $\cdots$ \\
\hline Effective Temper- & $5500 \pm 100$ & $6030 \pm 120$ & $\mathrm{~K}$ \\
$\quad$ & & & \\
ature $T_{\text {eff }}$ & $4.07 \pm 0.11$ & $4.07 \pm 0.10$ & $\mathrm{cgs}$ \\
Metallicity [Fe/H] & $0.01 \pm 0.11$ & $0.10 \pm 0.10$ & $\mathrm{dex}$ \\
$v$ sin $i_{\star}$ & $2.2 \pm 0.5$ & $4.6 \pm 0.5$ & $\mathrm{~km} \mathrm{~s}{ }^{-1}$ \\
Spectral Type & $\mathrm{G} 4 \mathrm{~V}$ & $\mathrm{~F} 9 \mathrm{IV}$ & $\cdots$ \\
\hline$B$ mag (UCAC4) & $13.56 \pm 0.01$ & $13.64 \pm 0.01$ & $\mathrm{mag}$ \\
$V$ mag (UCAC4) & $12.79 \pm 0.02$ & $12.92 \pm 0.01$ & $\mathrm{mag}$ \\
$J$ mag (2MASS) & $11.41 \pm 0.02$ & $11.56 \pm 0.02$ & $\mathrm{mag}$ \\
$H$ mag (2MASS) & $11.09 \pm 0.03$ & $11.26 \pm 0.03$ & $\mathrm{mag}$ \\
$K$ mag (2MASS) & $10.99 \pm 0.02$ & $11.21 \pm 0.02$ & $\mathrm{mag}$ \\
\hline
\end{tabular}

mean of the three independent determinations, using the error bars to calculate the weighting factor. The stellar parameters for both systems are listed in Table 3, along with the main identifiers and optical and near-infrared magnitudes.

\subsection{Joint Analysis of Photometric and Radial Velocity Measurements}

We used the Transit Light Curve Modeling (TLCM) code (Csizmadia et al. 2015; Csizmadia et al. in preparation) for the simultaneous analysis of the detrended light curves and radial velocity measurements. TLCM uses the Mandel \& Agol (2002) model to fit planetary transit light curves. The RV measurements are modeled with a Keplerian orbit. The fit is optimized using first a genetic algorithm and then a simulated annealing chain.

The fitted parameters are the semimajor axis $a / R_{*}$ and planet radius $R_{\mathrm{p}} / R_{*}$, both scaled to the radius of the star, the orbital inclination $i$, the limb-darkening coefficients $u_{+}=u_{1}+u_{2}$ and $u_{-}=u_{1}-u_{2}$, the radial velocity semi-amplitude $K$ and the systemic $\gamma$-velocity. The period $\left(P_{\text {orb }}\right)$ and epoch of mid-transit $\left(T_{0}\right)$ are allowed to vary slightly around the values determined already by the detection.

For K2-107, the model did not converge to the global minimum when leaving all nine parameters completely free, instead it seemed to converge to a broader local minimum. We thus first modeled the light curve, keeping the epoch and period, as well as the limb-darkening coefficients fixed using estimates from Claret \& Bloemen (2011). This gave us first estimates on the inclination, planet radius ratio, and semimajor axis. In a second step, we fitted all nine free parameters as for 
Table 4

Parameters From Light Curve and RV Data Analysis

\begin{tabular}{|c|c|c|c|}
\hline Parameter & $\mathrm{K} 2-60$ & K2-107 & Unit \\
\hline Orbital period $P_{\text {orb }}$ & $3.00265 \pm 0.00004$ & $3.31392 \pm 0.00002$ & days \\
\hline Transit epoch $T_{0}$ & $6928.0593 \pm 0.0007$ & $7304.5244 \pm 0.0002$ & $\mathrm{BJD}_{\mathrm{TDB}}-2450000$ \\
\hline Transit duration & $3.08 \pm 0.10$ & $3.19 \pm 0.45$ & $\mathrm{hr}$ \\
\hline Semimajor axis $a$ & $0.045 \pm 0.003$ & $0.048 \pm 0.005$ & $\mathrm{au}$ \\
\hline Scaled planet radius $R_{\mathrm{P}} / R_{*}$ & $0.063 \pm 0.001$ & $0.083 \pm 0.001$ & $\cdots$ \\
\hline Orbital inclination angle $i$ & $88.49 \pm 0.96$ & $81.9 \pm 0.7$ & $\operatorname{deg}$ \\
\hline Limb-darkening coefficient $u_{-}$ & $0.29 \pm 0.10$ & $0.17 \pm 0.07$ & $\cdots$ \\
\hline Radial velocity semi-amplitude $K$ & $61.0 \pm 2.6$ & $95.5 \pm 1.3$ & $\mathrm{~m} \mathrm{~s}^{-1}$ \\
\hline Systemic radial velocity $\gamma$ & $-45.475 \pm 0.003$ & $-8.364 \pm 0.001$ & $\mathrm{~km} \mathrm{~s}^{-1}$ \\
\hline RV velocity offset between FIES and HARPS & $0.158 \pm 0.004$ & $\ldots$ & $\mathrm{km} \mathrm{s}^{-1}$ \\
\hline Eccentricity $e$ & 0 (fixed) & 0 (fixed) & $\cdots$ \\
\hline Stellar surface gravity $\log g_{\star}{ }^{\mathrm{a}}$ & $4.33 \pm 0.04$ & $4.05 \pm 0.07$ & $\operatorname{cgs}$ \\
\hline Age & $10.0 \pm 3.0$ & $4.25 \pm 1.75$ & Gyr \\
\hline Planetary mass $M_{\mathrm{P}}$ & $0.426 \pm 0.037$ & $0.84 \pm 0.08$ & $M_{\text {Jup }}$ \\
\hline Planetary radius $R_{\mathrm{P}}$ & $0.683 \pm 0.037$ & $1.44 \pm 0.15$ & $R_{\mathrm{Jup}}$ \\
\hline Planetary mean density & $1.7 \pm 0.3$ & $0.35 \pm 0.1$ & $\operatorname{cgs}$ \\
\hline Planetary surface gravity $\log g_{p}$ & $3.35 \pm 0.06$ & $3.00 \pm 0.07$ & $\operatorname{cgs}$ \\
\hline Planetary calculated effective temperature & $1400 \pm 50$ & $1780 \pm 90$ & $\mathrm{~K}$ \\
\hline
\end{tabular}

Note.

a Derived from the light-curve modeling, effective temperature, metal content, and isochrones.

$\mathrm{K} 2-60 \mathrm{~b}$, but restricted the parameter space with the priors as given by our first fit. To verify our results, we also modeled the light curve with different fixed inclinations, leaving all other parameters free. This confirmed our result of a high impact parameter.

We also fit the data for non-circular orbits. The best-fitting eccentricity for $\mathrm{K} 2-60$ is $0.09 \pm 0.03$ with a $p$-value of 0.92 ; as for K2-107, we obtained $0.06 \pm 0.05$ with a $p$-value of 0.66 . The $p$-values were calculated following Lucy \& Sweeney (1971). Both $p$-values are larger than the 0.05 level of significance. We concluded that the RV measurements do not allow us to prefer the eccentric solutions over the circular ones and thus fixed the orbit eccentricities to zero. This assumption is reasonable given the fact that short-period orbits are expected to have circularized. Using the equations from Leconte et al. (2010), we calculated the tidal timescales for the eccentricity evolution of the two systems. $^{16}$ Assuming a modified tidal quality factor of $Q_{\star}^{\prime}=10^{6.5}$ for the stars and $Q_{\mathrm{p}}^{\prime}=10^{5.5}$ for the planets (Jackson et al. 2008), the timescales are $\sim 400$ and $\sim 25$ Myr for K2-60 and K2-107, respectively. These timescales are shorter than the estimated ages of the two host stars (Table 4).

We also fitted for radial velocity trends that might unveil the presence of additional orbiting companions in the systems. We obtained radial accelerations that are consistent with zero.

The best-fitting transit model and circular RV curve of K2$60 \mathrm{~b}$ are shown in Figures 5 and 6, along with the photometric

\footnotetext{
16 The rotation periods of the stars are estimated from the stellar radii and $v \sin i_{\star}$, assuming that the objects are seen equator-on.
}

and RV data. Results for K2-107b are displayed in Figures 7 and 8 . We checked our results by performing a joint fit to the photometric and RV data using the MCMC code pyaneti (O. Barragán 2017, in preparation). Following the same method outlined in Barragán et al. (2016), we set uninformative uniform priors in a wide range for each parameter and explored the parameter space with 500 chains. The final parameter estimates are consistent within $1 \sigma$ with those obtained using TLCM.

From the results of the spectral analysis and joint data modeling, we used Yonsei-Yale (Yi et al. 2001; Demarque et al. 2004) and Dartmouth (Dotter et al. 2008) isochrones to estimate masses, radii, and ages of K2-60 and K2-107. We obtained results that are in agreement regardless of the adopted set of isochrones. For the final results, we used the Yonsei-Yale isochrones (Yi et al. 2001; Demarque et al. 2004). From the fundamental parameters of the host stars, we calculated radii and masses of the two transiting planets. The parameter estimates are listed in Table 4 for both systems.

\section{Discussion and Summary}

\section{1. $K 2-60 b$}

$\mathrm{K} 2-60 \mathrm{~b}$ is a transiting sub-Jovian planet with an orbital period of $3.00267 \pm 0.00006$ days. It orbits a G4 main-sequence star. The planet's calculated effective temperature is $1400 \pm 50 \mathrm{~K}$. With a radius of $0.683 \pm 0.037 R_{\text {Jup }}$ and a mass of $0.426 \pm 0.037 M_{\mathrm{Jup}}$, it is more dense than expected. The radius 


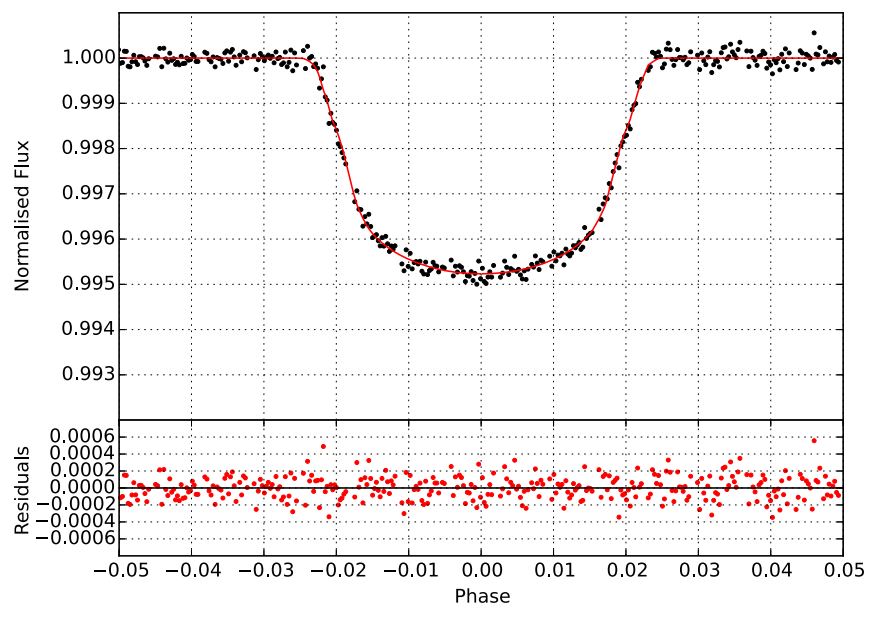

Figure 5. Phase folded light curve and best-fitting transit model (red line) of K2-60b. Residuals to the fit are shown in the lower panel.

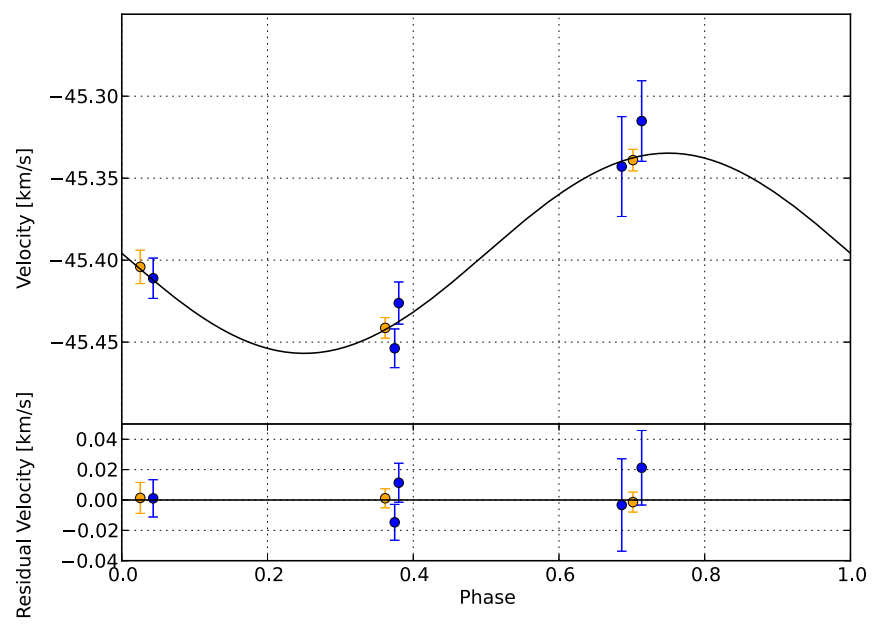

Figure 6. FIES (blue circles) and HARPS-N (orange circles) RV measurements of K2-60b and best-fitting circular model. Residuals to the fit are shown in the lower panel.

anomaly, based on the difference between model estimated to observed radius as described in Laughlin et al. (2011), is -0.46 , making this planet more dense than expected. Adaptive optics imaging by Schmitt et al. (2016) shows that there is no light contamination that could cause an underestimation of the planetary radius. We can exclude ellipsoidal variation with amplitudes above $0.05 \mathrm{mmag}$ in the light curve. There is no obvious trend in the radial velocity data, though we cannot exclude radial accelerations lower than $0.002 \mathrm{~km} \mathrm{~s}^{-1}$ day $^{-1}$.

The short orbital period and high effective temperature of the planet, along with its sub-Jovian size, put K2-60b close to the the so-called sub-Jovian desert. Figure 9 shows the known transiting planets with their radii plotted against their calculated effective temperatures as given by the equation in Laughlin et al. (2011)

$$
T_{\text {eff }}=\left(\frac{R_{S}}{2 a}\right)^{1 / 2} \frac{T_{S}}{\left(1-e^{2}\right)^{1 / 8}} .
$$

There is a clear lack of hot sub-Jovian planets. Due to different observational biases of exoplanet surveys (e.g., most of the inflated hot Jupiters have been detected by ground-based

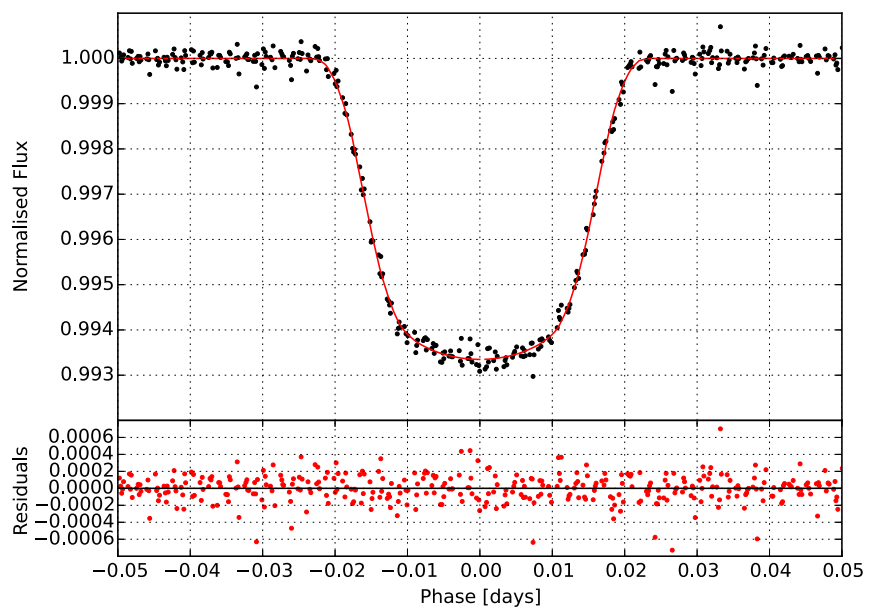

Figure 7. Phase folded light curve and the best-fitting transit model (red line) of K2-107b. Residuals to the fit are shown in the lower panel.

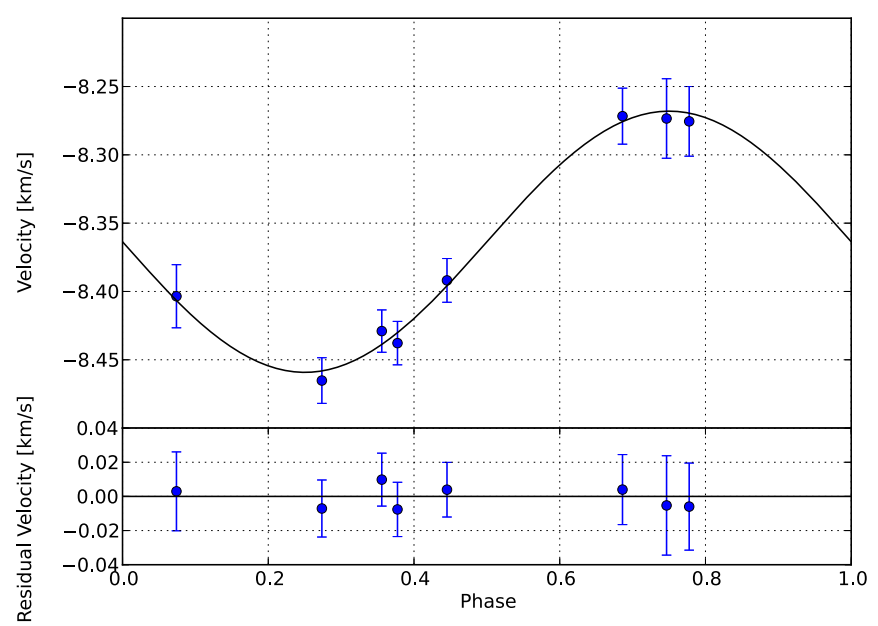

Figure 8. FIES RV measurements of K2-107b and best-fitting circular model Residuals to the fit are shown in the lower panel.

surveys, which might not be able to detect sub-Jovian planets with the same efficiency) the upper border is not as well defined as it may seem. This can be seen by looking only at confirmed planets of the Kepler spacecraft (blue points in Figure 9. Nevertheless, all observations suggest that the subJovian desert exists, though its borders are not well defined. Only a few planets are known in this regime (e.g., Sato et al. 2005; Bonomo et al. 2014). K2-60b might help in the future to get better restrictions on its borders.

\section{2. $K 2-107$}

K2-107b is a Jovian planet on a short orbital period of $3.31392 \pm 0.00002$ days. The planet orbits an F9 star about to leave the main sequence. It is one of only a few transiting planets known to orbit sub-giants (e.g., Almenara et al. 2015; Pepper et al. 2016; Smith et al. 2016; Van Eylen et al. 2016). The planet's calculated effective temperature is $1780 \pm 90 \mathrm{~K}$, its radius is $1.44 \pm 0.15 R_{\text {Jup }}$, and its mass is $0.84 \pm 0.08 M_{\text {Jup }}$. The radius anomaly is +0.21 , making $\mathrm{K} 2-107 \mathrm{~b}$ in contrast to $\mathrm{K} 2-60 \mathrm{~b}$, a highly inflated gaseous planet (see Figure 9, right). Such high inflation has already been observed for other giant planets with a similar effective temperature (see Figure 9). As suggested by 

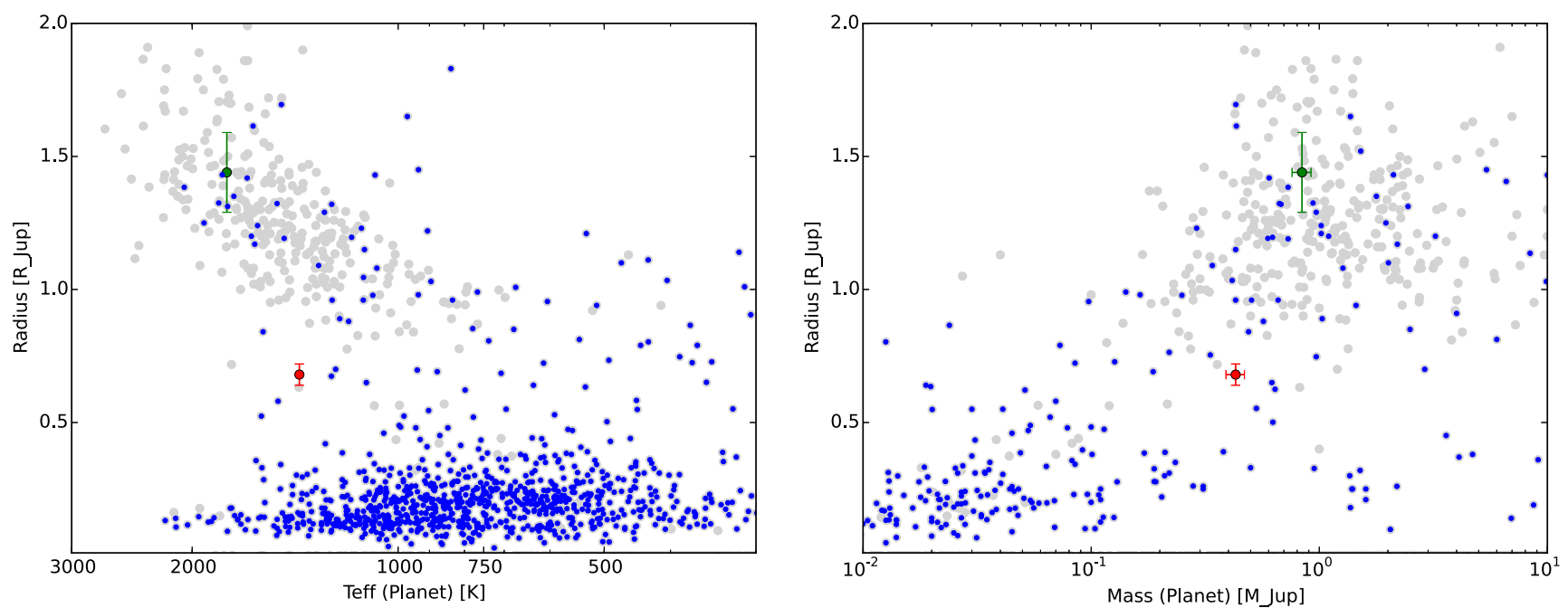

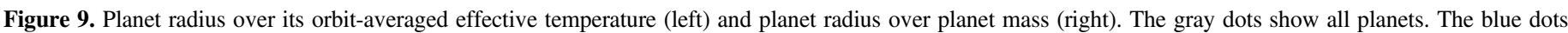

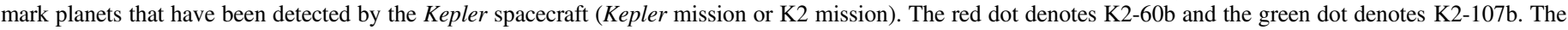
exoplanet data are taken from Extrasolar Planets Encyclopaedia (www.exoplanets.eu).

Laughlin et al. (2011), ohmic heating might be at least partly responsible for such inflation of the planet.

Since it is projected against the galactic center, K2-107 is in a relatively crowded stellar region. Using seeing-limited imaging and the GAIA public archive (DR1), we identify two faint stars within $\sim 10^{\prime \prime}$. The resulting contamination factor of 0.005 has been taken into account when modeling the light curve. The radial velocity data do not show any significant eccentricity or long-term trend higher than $0.001 \mathrm{~km} \mathrm{~s}^{-1}$ day $^{-1}$. The light curve of K2-107 shows no ellipsoidal variation with an amplitude larger than $0.1 \mathrm{mmag}$.

We would like to express our deepest gratitude to the NOT and TNG staff members for their unique support during the observations and scheduling of our runs. Szilard Csizmadia thanks the Hungarian OTKA Grant K113117. Hans Deeg and David Nespral acknowledge support by grant ESP2015-65712C5-4-R of the Spanish Secretary of State for R\& D\&i (MINECO). This research was supported by the Ministerio de Economia y Competitividad under project FIS2012-31079. The research leading to these results has received funding from the European Union Seventh Framework Programme (FP7/ 2013-2016) under grant agreement No. 312430 (OPTICON) and from the NASA K2 Guest Observer Cycle 1 program under grant NNX15AV58G to The University of Texas at Austin. Based on observations obtained with the Nordic Optical Telescope (NOT), operated on the island of La Palma jointly by Denmark, Finland, Iceland, Norway, and Sweden, in the Spanish Observatorio del Roque de los Muchachos (ORM) on the island of La Palma, and with the Italian Telescopio Nazionale Galileo (TNG) operated also at the ORM (IAC) by the INAF-Fundación Galileo Galilei. The data presented here were obtained in part with ALFOSC, which is provided by the Instituto de Astrofisica de Andalucia (IAA) under a joint agreement with the University of Copenhagen and NOTSA. This work has made use of data from the European Space Agency (ESA) mission Gaia (http://www.cosmos.esa.int/ gaia), processed by the Gaia Data Processing and Analysis Consortium (DPAC, http://www.cosmos.esa.int/web/gaia/ dpac/consortium). Funding for the DPAC has been provided by national institutions, in particular the institutions participating in the Gaia Multilateral Agreement. We are happy to acknowledge the continued involvement with help and upgrades to the Spectroscopy Made Easy (SME) program package, by N. Piskunov and J. Valenti. S.M.E. makes use of the VALD database, operated at Uppsala University, the Institute of Astronomy RAS in Moscow, and the University of Vienna (Ryabchikova et al. 2015).

Facilities: NOT (FIES, ALFOSC), TNG (HARPS-N), Kepler (K2), GAIA.

Software: IDL, IRAF, SME, DOOp, FAMA, TLCM, pyaneti.

\section{References}

Alibert, Y., Mordasini, C., \& Benz, W. 2011, A\&A, 526, A63 Almenara, J. M., Damiani, C., Bouchy, F., et al. 2015, A\&A, 575, A71 Baranne, A., Queloz, D., Mayor, M., et al. 1996, A\&AS, 119, 373 Barragán, O., Grziwa, S., Gandolfi, D., et al. 2016, arXiv:1608.01165 Batygin, K., Bodenheimer, P. H., \& Laughlin, G. P. 2016, ApJ, 829, 114 Beaugé, C., \& Nesvorný, D. 2013, ApJ, 763, 12

Bonomo, A. S., Sozzetti, A., Lovis, C., et al. 2014, A\&A, 572, A2 Borucki, W. J., Koch, D., Basri, G., et al. 2010, Sci, 327, 977 Bruntt, H., Bedding, T. R., Quirion, P.-O., et al. 2010, MNRAS, 405, 1907 Buchhave, L. A., Bakos, G. A., Hartman, J. D., et al. 2010, ApJ, 720, 1118 Cabrera, J., Csizmadia, Sz., Erikson, A., Rauer, H., \& Kirste, S. 2012, A\&A, 548, A44

Cantat-Gaudin, T., Donati, P., Pancino, E., et al. 2014, A\&A, 562, A10 Castelli, F., \& Kurucz, R. L. 2004, arXiv:astro-ph/0405087

Claret, A., \& Bloemen, S. 2011, A\&A, 529, A75

Cosentino, R., Lovis, C., Pepe, F., et al. 2012, Proc. SPIE, 8446, 84461V Crossfield, I. J. M., Ciardi, D. R., Petigura, E. A., et al. 2016, ApJS, 226, 7 Csizmadia, Sz., Hatzes, A., Gandolfi, D., et al. 2015, A\&A, 584, A13

Demarque, P., Woo, J.-H., Kim, Y.-C., \& Yi, S. K. 2004, ApJS, 155, 667

Dotter, A., Chaboyer, B., Jevremović, D., et al. 2008, ApJS, 178, 89

Doyle, A. P., Davies, G. R., Smalley, B., Chaplin, W. J., \& Elsworth, Y. 2014 MNRAS, 444, 3592

Frandsen, S., \& Lindberg, B. 1999, in Astrophysics with the NOT, FIES: A high resolution Fiber fed Echelle Spectrograph for the NOT, ed. H. Karttunen \& V. Piirola, 71

Gaia Collaboration, Brown, A. G. A., Vallenari, A., et al. 2016, A\&A, in press, arXiv: 1609.04172

Gandolfi, D., Parviainen, H., Deeg, H. J., et al. 2015, A\&A, 576, A11

Grziwa, S., Gandolfi, D., Csizmadia, Sz., et al. 2016a, AJ, 152, 132

Grziwa, S., \& Pätzold, M. 2016b, arXiv:1607.08417

Grziwa, S., Pätzold, M., \& Carone, L. 2012, MNRAS, 420, 1045 
Heiter, U., Lind, K., Asplund, M., et al. 2015, PhyS, 90, 054010 Howell, S. B., Sobeck, C., Haas, M., et al. 2014, PASP, 126, 398 Jackson, B., Greenberg, R., \& Barnes, R. 2008, ApJ, 678, 1396 Johnson, M. C., Gandolfi, D., Fridlund, M., et al. 2016, AJ, 151, 171 Laughlin, G., Crismani, M., \& Adams, F. C. 2011, ApJL, 729, L7 Leconte, J., Chabrier, G., Baraffe, I., \& Levrard, B. 2010, A\&A, 516, A64

Lindegren, L., Lammers, U., Bastian, U., et al. 2016, arXiv:1609.04303

Lucy, L. B., \& Sweeney, M. A. 1971, AJ, 76, 544

Lundkvist, M. S., Kjeldsen, H., Albrecht, S., et al. 2016, NatCo, 7, 11201

Magrini, L., Randich, S., Friel, E., et al. 2013, A\&A, 558, A38

Mandel, K., \& Agol, E. 2002, ApJL, 580, L171

Matsakos, T., \& Königl, A. 2016, ApJL, 820, L8

Mazeh, T., Holczer, T., \& Faigler, S. 2016, A\&A, 589, A75

Mordasini, C., Alibert, Y., \& Benz, W. 2009a, A\&A, 501, 1139

Mordasini, C., Alibert, Y., Benz, W., Klahr, H., \& Henning, T. 2012, A\&A, 541, A97

Mordasini, C., Alibert, Y., Benz, W., \& Naef, D. 2009b, A\&A, 501, 1161

Pepe, F., Mayor, M., Galland, F., et al. 2002, A\&A, 388, 632

Pepper, J., Rodriguez, J. E., Collins, K. A., et al. 2016, arXiv:1607.01755

Queloz, D., Henry, G. W., Sivan, J. P., et al. 2001, A\&A, 379, 279
Ryabchikova, T., Piskunov, N., Kurucz, R. L., et al. 2015, PhyS, 90, 054005 Ryabchikova, T. A., Pakhomov, Y. V., \& Piskunov, N. E. 2011, KIzKU, 153, 61

Sato, B., Fischer, D. A., Henry, G. W., et al. 2005, ApJ, 633, 465

Schmitt, J. R., Tokovinin, A., Wang, J., et al. 2016, AJ, 151, 159

Schneider, J., Dedieu, C., Le Sidaner, P., Savalle, R., \& Zolotukhin, I. 2011, A\&A, 532, A79

Smith, A. M. S., Gandolfi, D., Barragán, O., et al. 2016, arXiv:1609.00239

Sneden, C., Bean, J., Ivans, I., Lucatello, S., \& Sobeck, J. 2012, MOOG: LTE line analysis and spectrum synthesis, Astrophysics Source Code Library, ascl:1202.009

Stetson, P. B., \& Pancino, E. 2008, PASP, 120, 1332

Szabó, G. M., \& Kiss, L. L. 2011, ApJL, 727, L44

Telting, J. H., Avila, G., Buchhave, L., et al. 2014, AN, 335, 41

Toner, C. G., \& Gray, D. F. 1988, ApJ, 334, 1008

Udry, S., Mayor, M., \& Queloz, D. 1999, ASPC, 185, 367

Valenti, J. A., \& Fischer, D. A. 2005, ApJS, 159, 141

Valenti, J. A., \& Piskunov, N. 1996, A\&AS, 118, 595

Van Eylen, V., Albrecht, S., Gandolfi, D., et al. 2016, arXiv:1605.09180

Vanderburg, A., \& Johnson, J. A. 2014, PASP, 126, 948

Yi, S., Demarque, P., Kim, Y.-C., et al. 2001, ApJS, 136, 417 